\title{
LINEAR STABILITY ANALYSIS FOR FERROMAGNETIC FLUIDS IN THE PRESENCE OF MAGNETIC FIELD, COMPRESSIBILITY, INTERNAL HEAT SOURCE AND ROTATION THROUGH A POROUS MEDIUM
}

\author{
KAPIL Kumar \\ Department of Mathematics and Statistics, Gurukula Kangri Vishwavidyalaya, Haridwar, India \\ e-mail: kkchaudhary000@gmail.com \\ V. SingH \\ Department of Applied Sciences, Moradabad Institute of Technology, Moradabad, India \\ SEema SHARMA \\ Department of Mathematics and Statistics, Gurukula Kangri Vishwavidyalaya, Haridwar, India
}

\begin{abstract}
The effects of magnetic field and heat source strength on thermal convection of a compressible rotating ferromagnetic fluid through a porous medium are investigated theoretically using linear stability theory. A normal mode analysis method is employed to find solutions for the fluid layer confined between parallel planes with free boundaries. The cases of stationary and oscillatory instabilities are discussed. For the stationary state, compressibility, medium porosity and temperature gradient due to heat source have destabilizing effects, whereas rotation and ratio of magnetic permeability delay the onset of convection. The magnetic field and medium permeability have both stabilizing and destabilizing effects under certain conditions. The variations in the stationary critical thermal Rayleigh number and neutral instability curves in $\left(\mathrm{Ra}_{1}, x\right)$-plane for various values of physical parameters are shown graphically to depict the stability characteristics. The sufficient conditions for the non-existence of overstability are obtained and the principle of exchange of stabilities holds true in the absence of magnetic field and rotation under certain conditions.
\end{abstract}

Keywords: ferrofluids, rotation, magnetic field, porous medium, heat source, compressibility

\section{Introduction}

Ferrofluids (also known as magnetic fluids) are electrically non-conducting colloidal suspensions of fine solid ferromagnetic particles or nanoparticles (iron, nickel, cobalt etc.) and their study opens a wide range of attractive and futuristic applications in various engineering and medical science purposes like vacuum technology, instrumentation, lubrication mechanism, acoustics theory, recovery of metals, detection of tumours, drug delivery to a target site, magnetic fluid bearings, non-destructive testing, sensors and actuators, sorting of industrial scrap metals. They also serve as a challenging subject for scientists interested in the basics of fluid mechanics. The ferromagnetic nanoparticles are coated with a surfactant to prevent their agglomeration. Rosensweig $(1985,1987)$ discussed the fundamental concepts related to the use of ferrofluids and provides a comprehensive and detailed application of ferrohydrodynamics (also known as FHD) in various commercial usages such as novel zero-leakage rotary shaft seals used in computer disk drives (Bailey, 1983); semiconductor manufacturing (Moskowitz, 1975); pressure seals for compressor and blowers (Rosensweig, 1985); tracer of blood flow in non-invasive circulatory measurements (Newbower, 1972) and in loudspeakers to conduct heat away from the speakers coil (Hathaway, 1979). The thermal instability problem of ferrofluids is a current topic of frontier research and also attractive from a theoretical point of view. Thus, the overall field of ferrofluid research has a highly interdisciplinary character bringing physicists, engineers, chemists and 
mathematicians together. Finlayson (1970) discussed the convective instability problem of a ferromagnetic fluid layer heated from below when under the effect of a uniform vertical magnetic field with or without considering the effect of body force (gravity force). He quantified that the magnetization of a ferromagnetic fluid depends upon the magnetic field strength, temperature gradient and density of fluid, and is known as ferroconvection (which is very similar to Bénard convection as noted by Chandrasekhar, 1981). Lalas and Carmi (1971) studied a thermoconvective instability problem of ferrofluids without considering buoyancy effects, whereas the problem of thermal convection in a ferromagnetic fluid saturating a porous medium under the influence of rotation and/or suspended dust particles was simulated by Sunil et al. (2005a,b). Copious literatures (Odenbach, 2002; Neuringer and Rosensweig, 1964; Berkovsky and Bashtovoy, 1996; Sherman and Sutton, 1962) are available to deal with the hydrodynamic and hydromagnetic instability problems of ferrofluids and forcing further investigation in the whole research area.

The thermo-convective transport phenomenon in a rotating porous medium is of significant importance in modern science and engineering problems such as rotating machinery, crystal growth, food processing engineering, centrifugal filtration processes, biomechanics and in thermal power plants (to generate electricity by rotation of turbine blades). Magneto-hydrodynamics (MHD) theory of electrically conducting fluids has several scientific and practical applications in atmospheric physics, astronomy and astrophysics, space sciences, etc. Magnetic field is also used in several clinical areas such as neurology and orthopaedics for probing and curing the internal organs of the body in several diseases like tumours detection, heart and brain diseases, stroke damage, etc. Aggarwal and Makhija (2014) studied the effect of Hall current on thermal instability of ferromagnetic fluid in the presence of horizontal magnetic field through a porous medium. Spiegel and Veronis (1960) simplified the set of equations for compressible fluids by assuming that the vertical height of the fluid is much smaller than the scale height as defined by them, and the fluctuations in density, temperature and pressure did not exceed their total static variations. The thermal instability problem for a compressible fluid in the presence of rotation and magnetic field was studied by Sharma (1997).

Detailed investigations related with the problem of convection through various porous mediums were supplied and very well defined by Nield and Bejan (2006). The fluid flow problems saturating a porous medium plays a key role in petroleum and chemical industry, geophysical fluid dynamics, filtering technology, recovery of crude oil from Earth's interior, etc. Kumar et al. (2014a,b, 2015) addressed theoretically the thermal instability problems of couple-stress and ferromagnetic fluids by considering the effects of various parameters such as rotation, suspended particles, compressibility, heat source and variable gravity through Darcy and/or Brinkman porous medium. The physical properties of comets, meteorites and interplanetary dust strongly suggest the importance of porosity in astrophysical situations (McDonnel, 1978). The governing hydrodynamic equations of motion are solved using a regular perturbation technique. The objective of the present study is to discuss the influence of rotation, compressibility and heat source on thermal stability of a ferromagnetic fluid layer heated from below through a porous medium using linear stability analysis. The understanding of rotating ferrofluid instability problems plays a key role in microgravity environmental applications. Some existing results are recovered as a particular case of the present study.

\section{Governing equations}

Consider an infinite horizontal porous layer saturated with a non-conducting compressible ferromagnetic fluid confined between the parallel planes $z=0$ and $z=d$ subject to a uniform vertical magnetic field of intensity $\mathbf{H}(0,0, H)$ and uniform vertical rotation $\boldsymbol{\Omega}(0,0, \Omega)$. A Cartesian frame of reference is chosen with the $z$-axis directed vertically upwards and the $x$ - and $y$-axes at the lower boundary plane. It is also assumed that the flow in the porous medium is governed by 
Darcy's law in the equation of motion with medium porosity $\varepsilon$ and permeability $k_{1}$ for the case of free and perfect conducting boundaries. The geometrical configuration of the present problem is shown in Fig. 1.

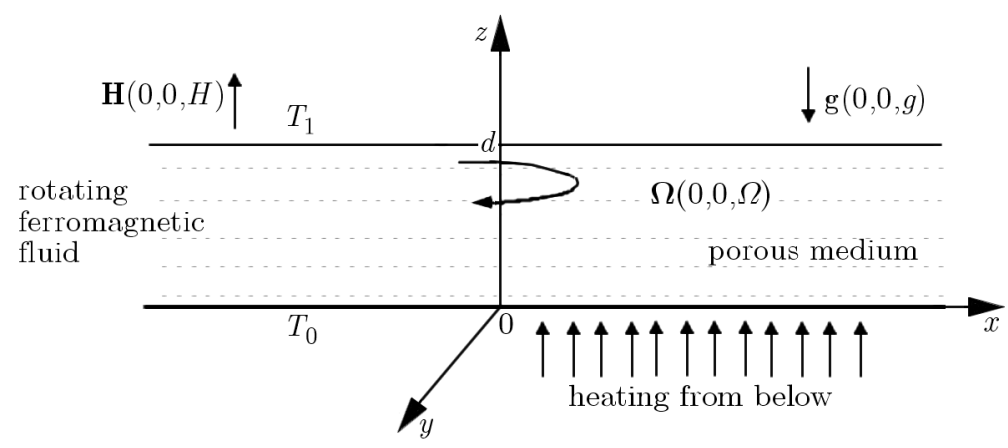

Fig. 1. Geometrical sketch of the physical problem

The basic governing equations of motion, continuity, energy and Maxwell equations for a magnetized ferrofluid saturating a homogenous porous medium with constant viscosity under Boussinesq approximation are given as follows (Finlayson, 1970; Rosensweig, 1985; Sunil et al., 2005a,b)

$$
\begin{aligned}
& \frac{\rho}{\varepsilon}\left[\frac{\partial \mathbf{q}}{\partial t}+\frac{1}{\varepsilon}(\mathbf{q} \cdot \nabla) \mathbf{q}\right]=-\nabla p+\rho \mathbf{X}_{i}+\mu_{0}(\mathbf{M} \cdot \nabla) \mathbf{H}-\left(\frac{\mu}{k_{1}}\right) \mathbf{q}+\frac{2 \rho}{\varepsilon}(\mathbf{q} \times \mathbf{\Omega}) \\
& \quad+\frac{\mu_{e}}{4 \pi}[(\nabla \times \mathbf{H}) \times \mathbf{H}] \\
& \varepsilon \frac{\partial \rho}{\partial t}+\nabla \cdot(\rho \mathbf{q})=0 \\
& {\left[\varepsilon \rho c_{v}+\rho_{s} c_{s}(1-\varepsilon)\right] \frac{\partial T}{\partial t}+\rho c_{v}(\mathbf{q} \cdot \nabla) T=k_{T} \nabla^{2} T+\Phi} \\
& \varepsilon\left(\frac{\partial \mathbf{H}}{\partial t}\right)=[\nabla \times(\mathbf{q} \times \mathbf{H})]+\varepsilon \eta\left(\nabla^{2} \mathbf{H} \quad \nabla \cdot \mathbf{H}=0\right.
\end{aligned}
$$

where the symbols $\rho, t, \mu, \mathbf{q}, \nabla p, \mu_{e}, \mu_{0}, \mathbf{H}, \mathbf{X}_{i}=-g \boldsymbol{\lambda}_{i}, \rho_{s}, c_{s}, c_{v}, T, k_{T}, \Phi$ and $\eta$ denote, respectively, density of the compressible fluid, time, co-efficient of viscosity, fluid velocity, pressure gradient term, magnetic permeability of the medium, magnetic permeability of vacuum $4 \pi \cdot 10^{-7} \mathrm{H} / \mathrm{m}$ ( $\mathrm{H}-$ Henry), magnetic field intensity, gravitational acceleration term, density of the solid material, heat capacity of the solid material, specific heat at constant volume, temperature, effective thermal conductivity, internal heat source strength and electrical resistivity.

The rotational effect induces two terms in the equation of motion, namely, the Centrifugal force $\left(-0.5 \operatorname{grad}|\boldsymbol{\Omega} \times \mathbf{r}|^{2}\right)$ and the Coriolis force $2(\mathbf{q} \times \boldsymbol{\Omega})$. In Eq. $(2.1)_{1}, p=\left(p_{f}-0.5 \rho|\boldsymbol{\Omega} \times \mathbf{r}|^{2}\right)$ is the reduced pressure, where $p_{f}$ stands for the fluid pressure and $\boldsymbol{\Omega}$ denotes the angular velocity.

Maxwell's equations for an electrically non-conducting fluid with no displacement currents are

$$
\nabla \cdot \mathbf{B}=0 \quad \nabla \times \mathbf{H}=\mathbf{0}
$$

The magnetic induction $\mathbf{B}$, magnetization $\mathbf{M}$ and the intensity of magnetic field $\mathbf{H}$ are related by (Penfield and Haus, 1967)

$$
\mathbf{B}=\mu_{0}(\mathbf{H}+\mathbf{M})
$$

In general, the magnetization $\mathbf{M}$ of a ferrofluid depends upon the magnitude of magnetic field $\mathbf{H}$ and temperature $T$, but in the present study it is assumed that the magnetization does not 
depend upon the magnetic field strength and is a function of temperature only. So, the magnetic equation of state takes the form

$$
\mathbf{M}=\mathbf{M}_{0}\left[1+\chi\left(T_{0}-T\right)\right]
$$

where $T_{0}$ and $\mathbf{M}_{0}$ are the reference temperature and reference magnetization, respectively, with $\mathbf{M}_{0}=\mathbf{M}\left(T_{0}\right) \cdot \chi=-\left(1 / \mathbf{M}_{0}\right)(\partial \mathbf{M} / \partial T)_{\mathbf{H}_{0}}$ stands for the pyromagnetic co-efficient and $\mathbf{H}_{0}$ is the uniform magnetic field of the fluid layer when placed in an external magnetic field $\mathbf{H}=\mathbf{H}_{0}^{\text {ext }} \times \boldsymbol{\lambda}_{i}$, where $\boldsymbol{\lambda}_{i}$ is the unit vector in the vertical direction.

According to Spiegel and Veronis (1960), the equations for compressible fluids are equivalent to those for incompressible fluids if the static temperature gradient $\beta$ is replaced by the term $\left(\beta-g / c_{p}\right)$ and $f$ is defined as any of the state variable $(p, \rho, T)$ and is expressed in the form

$$
f(x, y, z, t)=f_{m}+f_{0}(z)+f^{\prime}(x, y, z, t)
$$

where $f_{m}$ is the constant space distribution of $f, f_{0}$ is the variation in the absence of motion, $f^{\prime}(x, y, z, t)$ stands for the fluctuations in $f$ resulting from motion of the fluid and $c_{p}$ stands for the specific heat at constant pressure.

The quantities of the basic state are given by

$$
\begin{aligned}
& \mathbf{q}=\mathbf{q}_{b}=[0,0,0] \quad p=p_{b}(z) \quad \rho=\rho_{b}(z)=\rho_{0}(1+\alpha \beta z) \\
& \mathbf{H}=\mathbf{H}_{b}\left(0,0, H_{z}\right) \quad \mathbf{M}=\mathbf{M}_{b}(z) \quad \beta=\frac{T_{0}-T_{1}}{d} \\
& T=T_{b}(z)=T_{0}-\left(\beta-\frac{g}{c_{p}}\right) z+\frac{\Phi}{2 \kappa}\left(z d-z^{2}\right) \quad \mathbf{H}_{0}+\mathbf{M}_{0}=\mathbf{H}_{0}^{e x t}
\end{aligned}
$$

and

$$
\begin{aligned}
& \rho=\rho_{m}\left[1-\alpha_{m}\left(T-T_{m}\right)+K_{m}\left(p-p_{m}\right)\right] \quad \alpha_{m}=-\left(\frac{1}{\rho} \frac{\partial \rho}{\partial T}\right)_{m} \\
& K_{m}=\left(\frac{1}{\rho} \frac{\partial \rho}{\partial p}\right)_{m} \quad p(z)=p_{m}-g \int_{0}^{z}\left(\rho_{0}+\rho_{m}\right) d z
\end{aligned}
$$

where $\rho_{0}$ and $T_{0}$ stands for the density and temperature of the fluid at the lower boundary, whereas $p_{m}$ and $\rho_{m}$ stand for a constant space distribution of pressure $p$ and density $\rho$, respectively. The subscript $b$ denotes the basic state, $\alpha$ is the coefficient of thermal expansion and $\beta$ denotes the basic temperature gradient.

Now, to analyze the stability of the basic state using the perturbation technique, infinitesimal perturbations are assumed around the basic state solutions of the following form

$$
\begin{array}{lccc}
\mathbf{q}=\mathbf{q}_{b}+\mathbf{q}^{\prime} & p=p_{b}(z)+p^{\prime} & T=T_{b}(z)+\theta \quad \rho=\rho_{b}(z)+\rho^{\prime} \\
\mathbf{M}=\mathbf{M}_{b}(\mathbf{z})+\mathbf{M}^{\prime}\left(m_{x}, m_{y}, m_{z}\right) & \mathbf{H}=\mathbf{H}_{b}+\mathbf{h}\left(h_{x}, h_{y}, h_{z}\right)
\end{array}
$$

where $\mathbf{q}^{\prime}(u, v, w), p^{\prime}, \theta, \rho^{\prime}, \mathbf{M}^{\prime}, \mathbf{h}\left(h_{x}, h_{y}, h_{z}\right)$ are the perturbations in velocity $\mathbf{q}$, pressure $p$, temperature $T$, density $\rho$, magnetization $\mathbf{M}$ and magnetic field intensity $\mathbf{H}$, respectively. The changes in density and magnetization $\mathbf{M}^{\prime}$ caused by perturbation $\theta$ in temperature $T$ are defined as

$$
\rho^{\prime}=-\alpha \rho_{m} \theta \quad \mathbf{M}^{\prime}=-\chi \mathbf{M}_{0} \theta
$$


Using equation (2.8) in equations (2.1) and assuming the perturbation quantities to be very small, the following linearized perturbation equations are obtained as follows

$$
\begin{array}{ll}
\frac{1}{\varepsilon}\left(\frac{\partial \mathbf{q}^{\prime}}{\partial t}\right)=-\frac{1}{\rho_{m}}\left(\nabla p^{\prime}\right)-\mathbf{g}\left(\frac{\rho^{\prime}}{\rho_{m}}\right) \boldsymbol{\lambda}_{i}-\frac{\mu_{0} \chi \mathbf{M}_{0}(\nabla \mathbf{H}) \theta}{\rho_{m}}+\frac{\mu_{0}(\mathbf{M} \cdot \nabla) \mathbf{h}}{\rho_{m}}-\frac{v}{k_{1}} \mathbf{q}^{\prime} \\
+\frac{2}{\varepsilon}\left(\mathbf{q}^{\prime} \times \mathbf{\Omega}\right)+\frac{\mu_{e}}{4 \pi \rho_{m}}[(\nabla \times \mathbf{h}) \times \mathbf{H}] \\
\nabla \cdot \mathbf{q}^{\prime}=0 \quad E\left(\frac{\partial \theta}{\partial t}\right)=-\left(\frac{\partial T_{b}}{\partial z}\right) w+\kappa\left(\nabla^{2} \theta\right) \\
\nabla \cdot \mathbf{h}=0 \quad \varepsilon\left(\frac{\partial \mathbf{h}}{\partial t}\right)=(\nabla \mathbf{H}) \mathbf{q}^{\prime}+\varepsilon \eta\left(\nabla^{2} \mathbf{h}\right)
\end{array}
$$

where $E=\varepsilon+(1-\varepsilon)\left[\rho_{s} c_{s} /\left(\rho_{m} c_{v}\right)\right], \boldsymbol{\lambda}_{i}=[0,0,1]$ and $\mathbf{w}$ stands for the vertical fluid velocity. Eliminating $u, v$ and $\nabla p^{\prime}$ from the momentum equation and retaining the vertical component of fluid velocity, the following perturbation equations are obtained

$$
\begin{array}{lr}
\frac{1}{\varepsilon} \frac{\partial}{\partial t}\left(\nabla^{2} \mathbf{w}\right)=\left(\mathbf{g} \alpha-\frac{\mu_{0} \chi \mathbf{M}_{0} \nabla \mathbf{H}}{\rho_{m}}\right] \nabla_{1}^{2} \theta+\frac{\mu_{0} \mathbf{M}_{0}(1+\chi \Delta T)}{\rho_{m}} \nabla_{1}^{2}\left(\frac{\partial \mathbf{h}_{z}}{\partial z}\right)-\frac{v}{k_{1}}\left(\nabla^{2} \mathbf{w}\right) \\
-\frac{2 \boldsymbol{\Omega}}{\varepsilon}\left(\frac{\partial \boldsymbol{\zeta}}{\partial z}\right)+\frac{\mu_{e} \mathbf{H}}{4 \pi \rho_{m}}\left[\frac{\partial}{\partial z}\left(\nabla^{2} \mathbf{h}_{z}\right)\right] & \left(E \frac{\partial}{\partial t}-\kappa \nabla^{2}\right) \theta=\beta L h(z) w \\
\frac{1}{\varepsilon}\left(\frac{\partial \boldsymbol{\zeta}}{\partial t}\right)=-\frac{v}{k_{1}} \boldsymbol{\zeta}+\frac{2 \boldsymbol{\Omega}}{\varepsilon}\left(\frac{\partial \mathbf{w}}{\partial z}\right)+\frac{\mu_{e} \mathbf{H}}{4 \pi \rho_{m}}\left(\frac{\partial \boldsymbol{\xi}}{\partial z}\right) & \varepsilon\left(\frac{\partial \boldsymbol{\xi}}{\partial t}\right)=\mathbf{H}\left(\frac{\partial \boldsymbol{\zeta}}{\partial z}\right)+\varepsilon \eta\left(\nabla^{2} \boldsymbol{\xi}\right) \\
\varepsilon\left(\frac{\partial \mathbf{h}_{z}}{\partial t}\right)=\mathbf{H}\left(\frac{\partial \mathbf{w}}{\partial z}\right)+\varepsilon \eta\left(\nabla^{2} \mathbf{h}_{z}\right) &
\end{array}
$$

where $\boldsymbol{\xi}=\frac{\partial \mathbf{h}_{y}}{\partial x}-\frac{\partial \mathbf{h}_{x}}{\partial y}$ ( $z$-components of current density), $\boldsymbol{\zeta}=\frac{\partial \mathbf{v}}{\partial x}-\frac{\partial \mathbf{u}}{\partial y}$ ( $z$-component of vorticity), $\nabla^{2}=\frac{\partial^{2}}{\partial x^{2}}+\frac{\partial^{2}}{\partial y^{2}}+\frac{\partial^{2}}{\partial z^{2}}$ (three dimensional Laplacian operator), $\nabla_{1}^{2}=\frac{\partial^{2}}{\partial x^{2}}+\frac{\partial^{2}}{\partial y^{2}}$ (two dimensional horizontal Laplacian operator), $L=1-\frac{1}{G}=1-\frac{\beta c_{p}}{g}$ (modified dimensionless compressibility parameter), $S=\frac{\Phi d}{2 \beta \kappa L}$ (dimensionless heat source parameter), $h(z)=1-S\left(1-\frac{2 z}{d}\right)$ (non-uniform temperature gradient) and $\kappa=\frac{k_{T}}{\rho_{m} c_{v}}$ (thermal diffusivity of the fluid).

\section{Normal modes and linear stability analysis}

The system of equations (2.11) can be solved by using the method of normal modes in which the perturbation quantities have solutions with dependence upon $x, y$ and $t$ of the following form

$$
\left[w, \theta, \zeta, h_{z}, \xi\right]=[W(z), \Theta(z), Z(z), K(z), X(z)] \exp \left[\mathrm{i}\left(k_{x} x+k_{y} y\right)+n t\right]
$$

where $k_{x}$ and $k_{y}$ are the horizontal wave numbers along the $x$ and $y$ directions, respectively, $k^{2}=k_{x}^{2}+k_{y}^{2}$ is a dimensionless resultant wave number and $n$ is the growth rate of harmonic disturbance. Infinitesimal perturbations of the state may either grow or damp depending upon the growth rate $n$. Substituting expression (3.1) into linearized differential equations (2.11) along with $z=z^{*} d, a=k d, \sigma=n d^{2} / v, D=\partial / \partial z^{*}$, the following non-dimensional form is obtained (after ignoring the asterisk)

$$
\begin{aligned}
& \left(\frac{\sigma}{\varepsilon}+\frac{1}{P_{l}}\right)\left(D^{2}-a^{2}\right) W(z)=-\left(g-\frac{\mu_{0} \chi M_{0} \nabla H}{\rho_{m} \alpha}\right) \frac{\alpha a^{2} d^{2} \Theta}{v} \\
& \quad-\left[\frac{\mu_{0} M_{0}(1+\chi \Delta T)}{\rho_{m}} \frac{a^{2} d}{v}-\frac{\mu_{e} H d}{4 \pi \rho_{m} v}\left(D^{2}-a^{2}\right)\right] D K-\frac{2 \Omega d_{3}}{\varepsilon v} D Z
\end{aligned}
$$


and

$$
\begin{array}{ll}
\left(\frac{\sigma}{\varepsilon}+\frac{1}{P_{l}}\right) Z=\frac{2 \Omega d}{\varepsilon v} D W+\frac{\mu_{e} H d}{4 \pi \rho_{m} v} D X & {\left[\left(D^{2}-a^{2}\right)-E p_{1} \sigma\right] \Theta=-\frac{\beta d^{2}}{\kappa} L h(z) W} \\
{\left[\left(D^{2}-a^{2}\right)-p_{2} \sigma\right] K=-\frac{H d}{\varepsilon \eta} D W} & {\left[\left(D^{2}-a^{2}\right)-p_{2} \sigma\right] X=-\frac{H d}{\varepsilon \eta} D Z}
\end{array}
$$

The dimensionless parameters in equations (3.2) and (3.3) are the thermal Prandtl number $\operatorname{Pr}_{1}=v / \kappa$, the magnetic Prandtl number $\operatorname{Pr}_{2}=v / \eta$ and the dimensionless medium permeability $P_{l}=k_{1} / d^{2}$.

The boundary conditions appropriate for the case of two free boundaries are defined as

$$
\left\{\begin{array}{l}
W=D^{2} W=D Z=\Theta=0 \quad \text { at } z=0 \text { and } z=1 \\
h_{x}, h_{y}, h_{z} \text { are continuous at the boundaries }
\end{array}\right.
$$

The solution to equations (3.2) and (3.3) satisfying boundary conditions (3.4) can be taken in the form

$$
W=W_{0} \sin (l \pi z) \quad l=1,2,3, \ldots
$$

where $W_{0}$ is a constant. The most suitable mode corresponds to $l=1$ (fundamental mode). Therefore, using solution (3.5) with $l=1$ into equations (3.2) and (3.3), the dispersion relation is obtained as follows (after eliminating $\Theta, X, Z$ and $K$ )

$$
\begin{aligned}
& (1+x)\left(1+x+i E \operatorname{Pr}_{1} \sigma_{i}\right)\left(1+x+i \operatorname{Pr}_{2} \sigma_{i}\right)=\operatorname{Ra}_{1} x \varepsilon P L h(z) \frac{1+x+i \sigma_{i} \operatorname{Pr}_{2}}{\varepsilon+i \sigma_{i} P} \\
& \quad-\frac{P Q_{1}}{\varepsilon+i \sigma_{i} P}[x \Gamma+(1+x)]\left(1+x+i \sigma_{i} E \operatorname{Pr}_{1}\right) \\
& \quad-T_{A_{1}} P^{2}\left(1+x+i \sigma_{i} E \operatorname{Pr}_{1}\right)\left(1+x+i \sigma_{i} \operatorname{Pr}_{2}\right)^{2} \frac{1}{1+x+i \sigma_{i} \operatorname{Pr}_{2}+Q_{1} P}
\end{aligned}
$$

where $\mathrm{Ra}_{F}$ is the thermal Rayleigh number for ferromagnetic fluids, $Q$ - Chandrasekhar number, $Q_{M}$ - modified Chandrasekhar number for ferromagnetic fluids, $\Gamma$ - ratio of magnetic permeability with magnetization to magnetic strength and $T_{A}$ - Taylor number

$$
\begin{array}{lll}
\operatorname{Ra}_{F}=\left(g-\frac{\mu_{0} \chi M_{0} \nabla H}{\rho_{m} \alpha}\right) \frac{\alpha \beta d^{4}}{v \kappa} & Q=\frac{\mu_{e} H^{2} d^{2}}{4 \pi \rho_{m} v \eta} & Q_{M}=\frac{\mu_{0} M_{0}(1+\chi \Delta T)}{\rho_{m}} \frac{H d^{2}}{v \eta} \\
\Gamma=\frac{Q_{M_{1}}}{Q_{M}}=\frac{4 \pi \mu_{0} M}{\mu_{e} H} & T_{A}=\frac{4 \Omega^{2} d^{4}}{v^{2}}
\end{array}
$$

and

$$
\begin{aligned}
& \mathrm{Ra}_{1}=\frac{\mathrm{Ra}_{F}}{\pi^{4}} \quad x=\frac{a^{2}}{\pi^{2}} \quad i \sigma_{i}=\frac{\sigma}{\pi^{2}} \quad P=\pi^{2} P_{l} \\
& Q_{1}=\frac{Q}{\pi^{2}} \quad Q_{M_{1}}=\frac{Q_{M}}{\pi^{2}} \quad T_{A_{1}}=\frac{T_{A}}{\pi^{4}}
\end{aligned}
$$

Equation (3.6) is the required dispersion relationship that accounts for the effects of rotation, medium permeability, medium porosity, compressibility, uniform heat source and magnetic field on thermal instability of the ferromagnetic fluid in a porous medium.

From equation (3.6), the thermal Rayleigh number $\mathrm{Ra}_{1}$ can be separated into the real and imaginary parts as

$$
\mathrm{Ra}_{1}=X_{1}+i \sigma_{i} X_{2}
$$


where $X_{1}, X_{2}$ and $\sigma_{i}$ are real numbers defined as

$$
\begin{aligned}
X_{1} & =\frac{1}{x \varepsilon P L h(z)}\left(\left[(1+x)^{2} \varepsilon-\sigma_{i}^{2} \operatorname{PPr}_{1} E(1+x)\right]\right. \\
& +\frac{P Q_{1}[x \Gamma+(1+x)]\left[(1+x)^{2}+\sigma_{i}^{2} \operatorname{Pr}_{1} \operatorname{Pr}_{2} E\right]}{(1+x)^{2}+\sigma_{i}^{2} \operatorname{Pr}_{2}^{2}} \\
& +\frac{T_{A_{1}} P^{2}}{\left[(1+x)+Q_{1} P\right]^{2}+\sigma_{i}^{2} \operatorname{Pr}_{2}^{2}}\left\{\left[(1+x)^{2}-\sigma_{i}^{2} \operatorname{Pr}_{1} \operatorname{Pr}_{2} E\right]\left[\left(1+x+Q_{1} P\right) \varepsilon+\sigma_{i}^{2} P \operatorname{Pr}_{2}\right]\right. \\
& \left.\left.-\sigma_{i}^{2}\left[(1+x)\left(\operatorname{Pr}_{2}+\operatorname{Pr}_{1} E\right)\right]\left[(1+x) P+Q_{1} P^{2}-\operatorname{Pr}_{2} \varepsilon\right]\right\}\right) \\
X_{2} & =\frac{1}{x \varepsilon P L h(z)}\left(\left[(1+x)^{2} P+\operatorname{Pr}_{1} E \varepsilon(1+x)\right]+\frac{P Q_{1}[x \Gamma+(1+x)](1+x)\left(\operatorname{Pr}_{1} E-\operatorname{Pr}_{2}\right)}{(1+x)^{2}+\sigma_{i}^{2} \operatorname{Pr}_{2}^{2}}\right. \\
& +\frac{T_{A_{1}} P^{2}}{\left[(1+x)+Q_{1} P\right]^{2}+\sigma_{i}^{2} \operatorname{Pr}_{2}^{2}}\left\{\left[(1+x)^{2}-\sigma_{i}^{2} \operatorname{Pr}_{1} \operatorname{Pr}_{2} E\right]\left[\left(1+x+Q_{1} P\right) P-\operatorname{Pr}_{2} \varepsilon\right]\right. \\
& \left.\left.+\left[\left(1+x+Q_{1} P\right) \varepsilon+\sigma_{i}^{2} \operatorname{PPr}_{2}\right](1+x)\left(\operatorname{Pr}_{2}+\operatorname{Pr}_{1} E\right)\right\}\right)
\end{aligned}
$$

Since $\mathrm{Ra}_{1}$ is a physical quantity, it must be real. Hence, from equation (3.7) it follows that either $\sigma_{i}=0$ (stationary state) or $X_{2}=0, \sigma_{i} \neq 0$ (oscillatory state). It should also be noted that when $\mu_{0}=0$ (i.e. $\Gamma=0$ ) then from equation (3.8) ${ }_{2} X_{2}$ cannot vanish and therefore, $\sigma_{i}$ must be zero. This implies that for an ordinary viscous fluid, the principle of exchange of stabilities is valid even in the presence of a porous medium, and this statement is verified in Section 6 .

\subsection{The stationary state}

For real $\sigma_{i}$, the marginal instability (or neutral instability) occurs when $\sigma_{i}=0$. Substituting $\sigma_{i}=0$ into equations (3.7) and (3.8) 1 , the modified thermal Rayleigh number is obtained for the onset of stationary convection in the following form

$$
\mathrm{Ra}_{1}^{\text {stat }}=\frac{1}{x \varepsilon P \operatorname{Lh}(z)}\left\{(1+x)^{2} \varepsilon+P Q_{1}[x \Gamma+(1+x)]+T_{A_{1}} P^{2} \frac{(1+x)^{2} \varepsilon}{Q_{1} P+(1+x)}\right\}
$$

Equation (3.9) leads to the marginal instability curves in stationary conditions.

For higher values of permeability $(P \rightarrow \infty)$ which correspond to the case of pure fluids, equation (3.9) gives

$$
\mathrm{Ra}_{1}^{\text {stat }}=\frac{1}{\operatorname{Lh}(z)}\left\{\frac{Q_{1}[x \Gamma+(1+x)]}{\varepsilon x}+\frac{T_{A_{1}}(1+x)^{2}}{Q_{1} x}\right\}
$$

Minimizing equation (3.9) with respect to $x$ yields an equation of degree four in $x$ as

$$
x^{4}+A_{1} x^{3}+A_{2} x^{2}+A_{3} x+A_{4}=0
$$

where

$$
\begin{aligned}
& A_{1}=2\left(1+Q_{1} P\right) \\
& A_{2}=Q_{1}^{2} P^{2}+2 Q_{1} P-\frac{Q_{1} P}{\varepsilon}+T_{A_{1}} Q_{1} P^{3}-T_{A_{1}} P^{2} \\
& A_{3}=-\left[2\left(1+Q_{1} P\right)+2 T_{A_{1}} P^{2}+\frac{2 Q_{1} P\left(1+Q_{1} P\right)}{\varepsilon}\right] \\
& A_{4}=-1+Q_{1}^{2} P^{2}+2 Q_{1} P-\frac{Q_{1} P}{\varepsilon}-T_{A_{1}} Q_{1} P^{3}-T_{A_{1}} P^{2}-\frac{\left(Q_{1}^{2} P^{2}+2 Q_{1} P\right) Q_{1} P}{\varepsilon}
\end{aligned}
$$


In the absence of heat source parameter (i.e. $h(z)=1$ ), equation (3.9) gives

$$
\mathrm{Ra}_{1}^{\text {stat }}=\frac{1}{x \varepsilon P L}\left\{(1+x)^{2} \varepsilon+P Q_{1}[x \Gamma+(1+x)]+T_{A_{1}} P^{2} \frac{(1+x)^{2} \varepsilon}{Q_{1} P+(1+x)}\right\}
$$

which agrees with the previous published work by Aggarwal and Makhija (2014) in the absence of the Hall effect but in the presence of rotation and compressibility.

The classical results for Newtonian fluids can be obtained as a particular case of the present study.

For an incompressible $(L=1)$, non-rotatory and non-magnetized system, equation (3.12) reduces to

$$
\mathrm{Ra}_{1}^{\text {stat }}=\frac{(1+x)^{2}}{P x}
$$

This coincides with the classical Rayleigh-Bénard result for a Newtonian fluid in a porous medium.

To analyze the effects of various parameters such as modified compressibility, medium porosity, temperature gradient due to internal heating, rotation, magnetic field and medium permeability, the behaviour of $d \mathrm{Ra}_{1}^{\text {stat }} / d L, d \mathrm{Ra}_{1}^{\text {stat }} / d \varepsilon, d \mathrm{Ra}_{1}^{\text {stat }} / d h(z), d \mathrm{Ra}_{1}^{\text {stat }} / d T_{A_{1}}, d \mathrm{Ra}_{1}^{\text {stat }} / d Q_{1}$ and $d \mathrm{Ra}_{1}^{\text {stat }} / d P$ is examined analytically.

Differentiating equation (3.9) with respect to various parameters, i.e. $L, \varepsilon, h(z), T_{A_{1}}, Q_{1}, P$, leads to following expressions

$$
\begin{aligned}
& \frac{d \mathrm{Ra}_{1}^{\text {stat }}}{d L}=-\frac{R^{\oplus}}{L^{2} h(z)} \quad \frac{d \mathrm{Ra}_{1}^{\text {stat }}}{d \varepsilon}=-\frac{1}{L h(z)}\left\{\frac{[x \Gamma+(1+x)] Q_{1}}{\varepsilon^{2} x}\right\} \\
& \frac{d \mathrm{Ra}_{1}^{\text {stat }}}{d h(z)}= \begin{cases}\frac{1}{L(1-S)^{2}} R^{\oplus} & \text { at } \quad z=0 \\
-\frac{1}{L(1+S)^{2}} R^{\oplus} & \text { at } \quad z=d\end{cases}
\end{aligned}
$$

This shows that the modified compressibility, medium porosity and temperature gradient (except for the lower boundary) have a destabilizing effect

$$
\frac{d \mathrm{Ra}_{1}^{\text {stat }}}{d T_{A_{1}}}=\frac{1}{\operatorname{Lh}(z)}\left\{\frac{P(1+x)^{2}}{x\left[Q_{1} P+(1+x)\right]}\right\}
$$

which is positive, thereby implying the stabilizing effect of the rotational parameter

$$
\begin{aligned}
\frac{d \mathrm{Ra}_{1}^{\text {stat }}}{d Q_{1}} & =\frac{1}{\operatorname{Lh}(z)}\left\{\frac{x \Gamma+(1+x)}{\varepsilon x}-\frac{T_{A_{1}} P^{2}(1+x)^{2}}{x\left[Q_{1} P+(1+x)\right]^{2}}\right\} \\
\frac{d \mathrm{Ra}_{1}^{\text {stat }}}{d P} & =\frac{1}{\operatorname{Lh}(z)}\left\{\frac{T_{A_{1}}(1+x)^{2}}{x\left[Q_{1} P+(1+x)\right]}-\frac{P Q T_{A_{1}}(1+x)^{2}}{x\left[Q_{1} P+(1+x)\right]^{2}}-\frac{(1+x)^{2}}{P^{2} x}\right\}
\end{aligned}
$$

Equations (3.16) show that the magnetic field and medium permeability have dual effects. In a non-rotating frame, the magnetic field has a stabilizing effect, whereas permeability has a destabilizing effect where

$$
R^{\oplus}=\frac{(1+x)^{2}}{P x}+\frac{[x \Gamma+(1+x)] Q_{1}}{\varepsilon x}+\frac{T_{A_{1}} P(1+x)^{2}}{x\left[Q_{1} P+(1+x)\right]}
$$




\subsection{The oscillatory state}

For an oscillatory state, setting $X_{2}=0, \sigma_{i} \neq 0$ in equation $(3.8)_{2}$ gives a polynomial in $\sigma_{i}^{2}$ of degree two in the form

$$
a_{0} \sigma_{i}^{4}+a_{1} \sigma_{i}^{2}+a_{2}=0
$$

Solving equation (3.17) for $\sigma_{i}^{2}$, one gets

$$
\sigma_{i}^{2}=\frac{-a_{1} \pm \sqrt{a_{1}^{2}-4 a_{0} a_{2}}}{2 a_{0}}
$$

For simplicity, the values of coefficients $a_{0}, a_{1}$ and $a_{2}$ are not mentioned here to save spaces.

With $\sigma_{i}^{2}$ determined from equation (3.18), the Rayleigh number for an oscillatory instability can be obtained with the help of equations (3.7) and (3.8) 1 as

$$
\begin{aligned}
\mathrm{Ra}_{1}^{\text {osc }} & =\frac{1}{x \varepsilon P L h(z)}\left((1+x)^{2} \varepsilon-\sigma_{i}^{2} \operatorname{PPr}_{1} E(1+x)\right. \\
& +\frac{P Q_{1}[x \Gamma+(1+x)]\left[(1+x)^{2}+\sigma_{i}^{2} \operatorname{Pr}_{1} \operatorname{Pr}_{2} E\right]}{(1+x)^{2}+\sigma_{i}^{2} \operatorname{Pr}_{2}^{2}} \\
& +\frac{T_{A_{1}} P^{2}}{\left[(1+x)+Q_{1} P\right]^{2}+\sigma_{i}^{2} \operatorname{Pr}_{2}^{2}}\left\{\left[(1+x)^{2}-\sigma_{i}^{2} \operatorname{Pr}_{1} \operatorname{Pr}_{2} E\right]\left[\left(1+x+Q_{1} P\right) \varepsilon+\sigma_{i}^{2} P \operatorname{Pr}_{2}\right]\right. \\
& \left.\left.-\sigma_{i}^{2}(1+x)\left(\operatorname{Pr}_{2}+\operatorname{Pr}_{1} E\right)\left[(1+x) P+Q_{1} P^{2}-\operatorname{Pr}_{2} \varepsilon\right]\right\}\right)
\end{aligned}
$$

The values of the critical wave number $x_{c}$ for the oscillatory case can be obtained from equation (3.19) with the condition $d \mathrm{Ra}_{1}^{\text {osc }} / d x=0$ and then substituting this critical wave number $x_{c}$ into equation (3.19) yields the critical Rayleigh number $\mathrm{Ra}_{1 c}^{o s c}$ for the oscillatory instability. Further, substituting these critical wave number and the critical Rayleigh number of oscillatory instability into equation (3.18) gives the critical frequency for the oscillatory case.

\section{Results and discussion}

In the present Section, we mainly focused on the determination of critical wave numbers and critical thermal Rayleigh numbers for the stationary case. The values of the critical wave number $x_{c}$ for the onset of stationary instability are determined numerically from equation (3.11) with the condition $d \mathrm{Ra}_{1}^{\text {stat }} / d x=0$, and then equation (3.9) will give the critical thermal Rayleigh number for the stationary state. The variations in critical thermal Rayleigh numbers $\mathrm{Ra}_{1 c}^{\text {stat }}$ for various values of physical parameters are depicted graphically in Fig. 2. Also, the variations of marginal (neutral) instability curves in the $\left(\mathrm{Ra}_{1}-x\right)$ plane for different parametric values $\left(L, h(z), \varepsilon, T_{A_{1}}, Q_{1}, P\right)$ are shown in Fig. 3. We fixed the values of the parameters except for the varying parameter. 
(a)

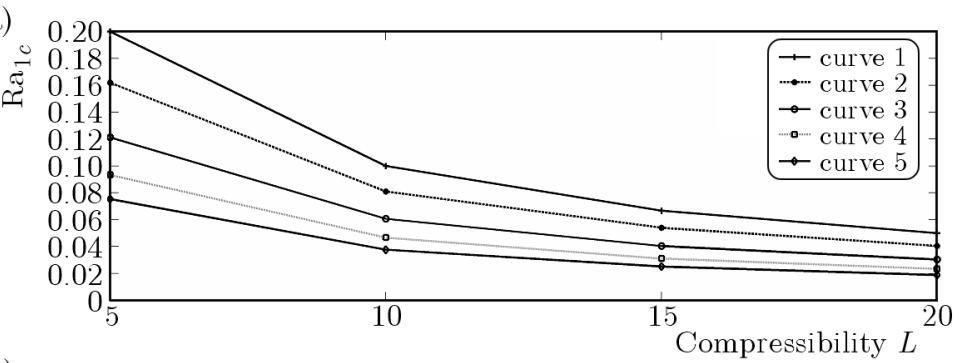

(b)

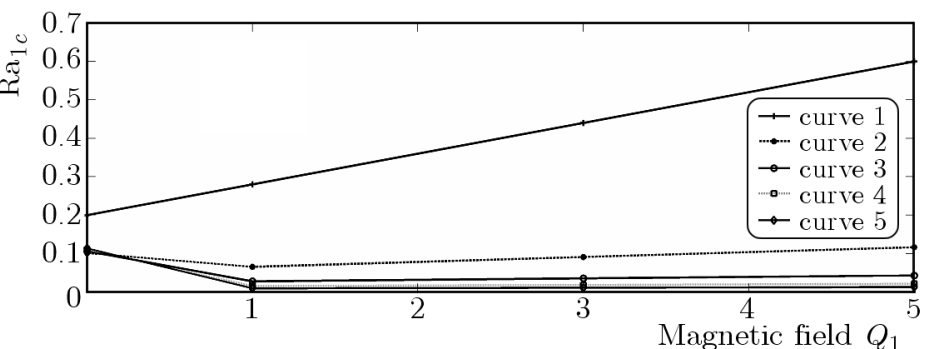

(c)

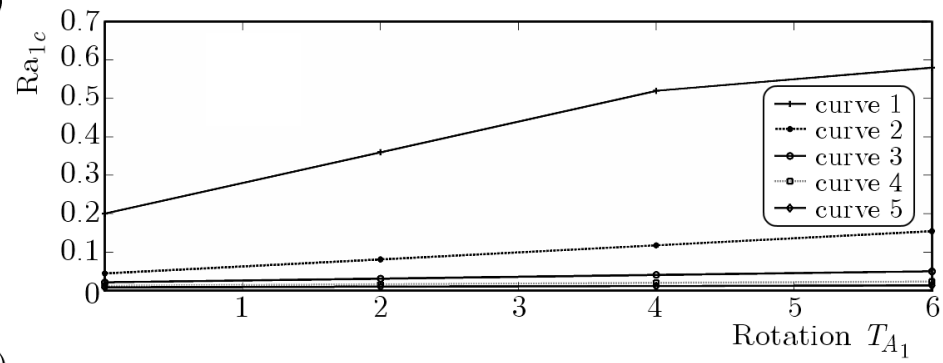

(d)

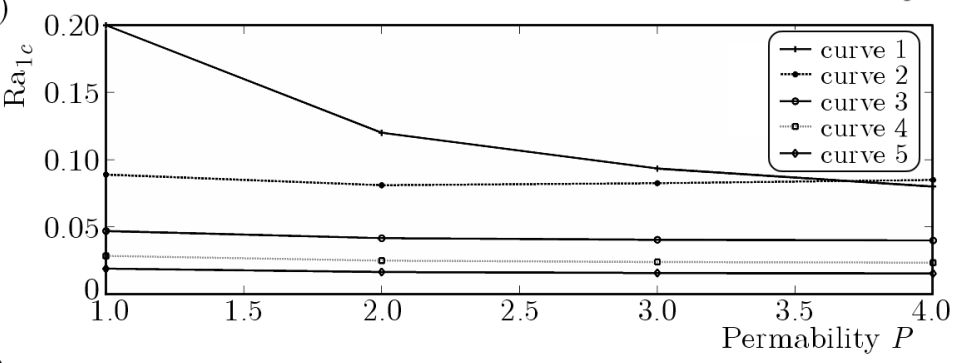

(e)

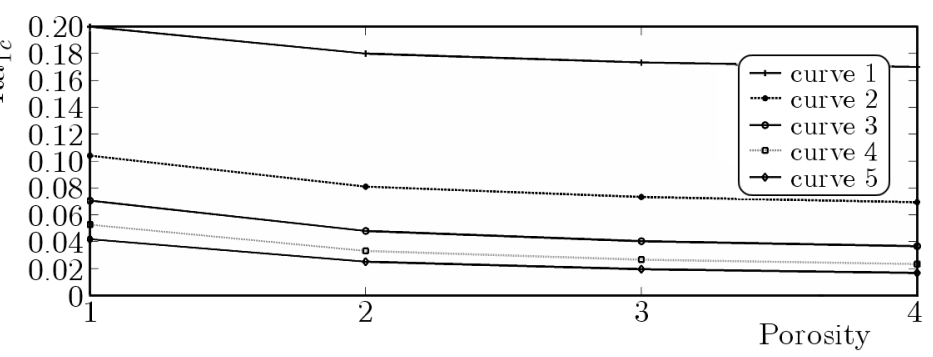

(f)

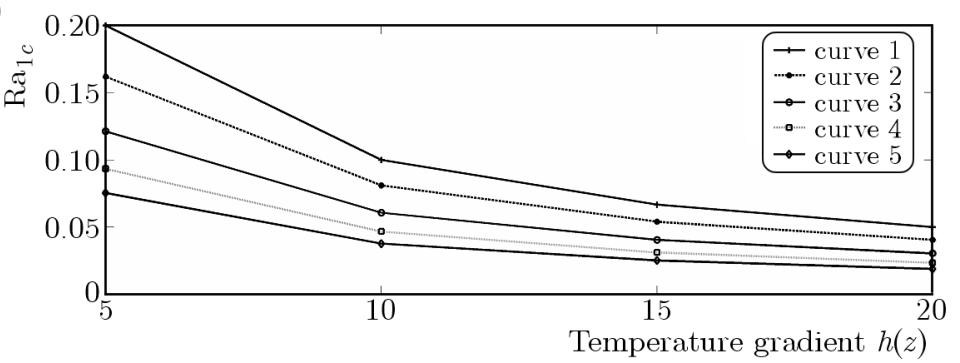

Fig. 2. Variation of $\mathrm{Ra}_{1 c}$ verrsus $L$ (a), $T_{A_{1}}$ (b), $Q_{1}$ (c), $P$ (d), $\varepsilon$ (e), $h(z)$ (f) various valuesof physical parameters; curve 1: $Q_{1}=0, Q_{M_{1}}=1, \varepsilon=1, P=1, h(z)=5, L=5, T_{A_{1}}=0$, curve $2: Q_{1}=1$, $Q_{M_{1}}=3, \varepsilon=2, P=2, h(z)=10, L=10, T_{A_{1}}=2$, curve $3: Q_{1}=3, Q_{M_{1}}=5, \varepsilon=3, P=3, h(z)=15$, $L=15, T_{A_{1}}=4$, curve 4: $Q_{1}=5, Q_{M_{1}}=7, \varepsilon=4, P=4, h(z)=20, L=20, T_{A_{1}}=6$, curve $5: Q_{1}=7$, $Q_{M_{1}}=9, \varepsilon=5, P=5, h(z)=25, L=25, T_{A_{1}}=8$ 
(a)

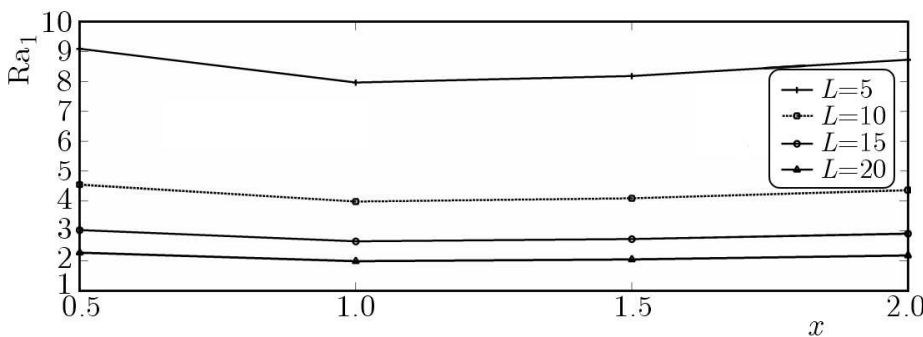

(b)

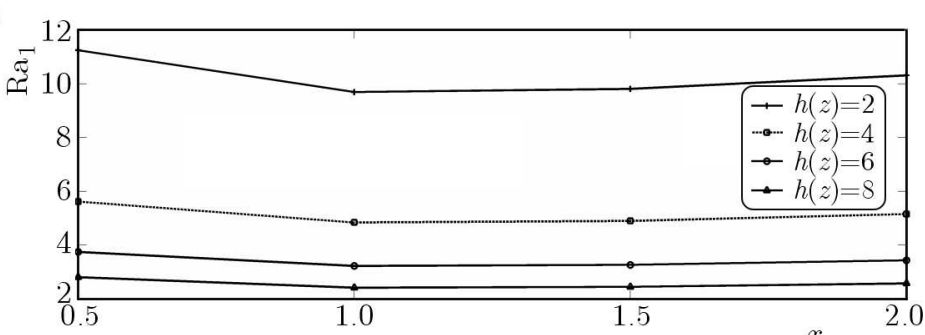

(c)

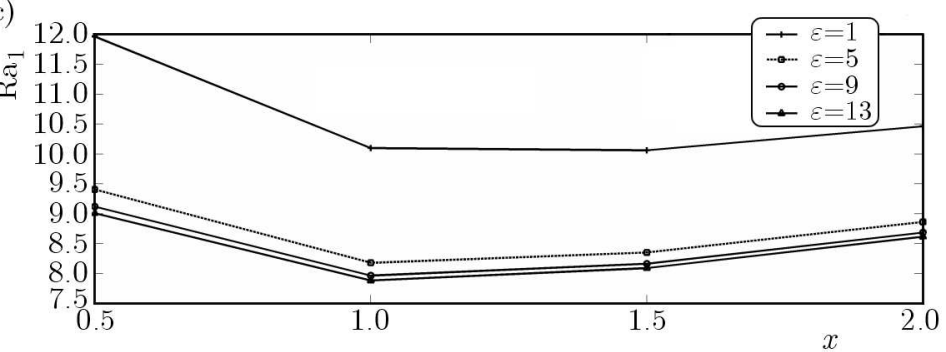

(d)

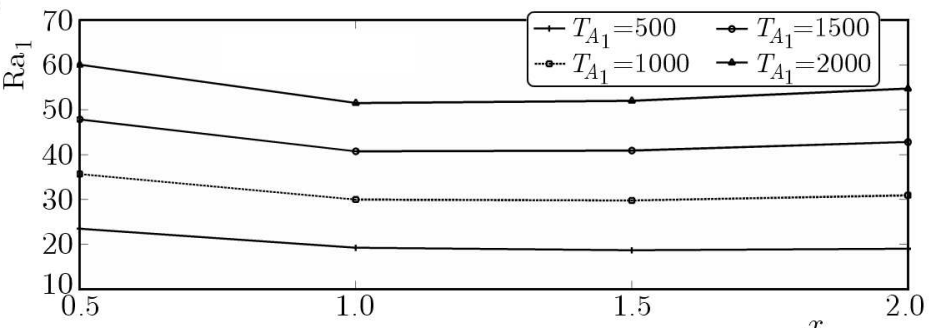

(e)

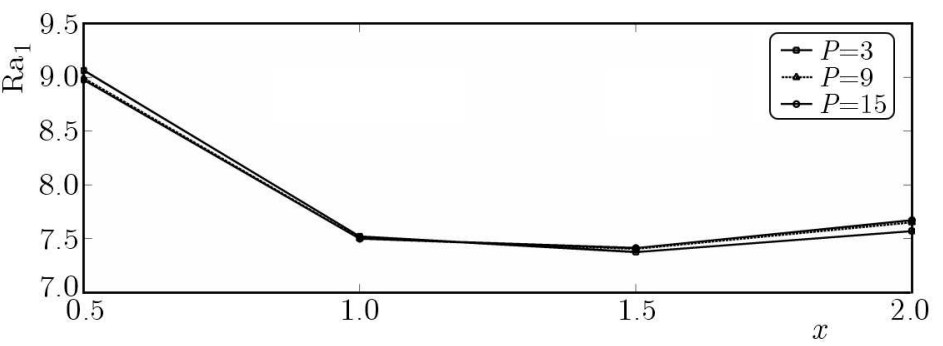

(f)

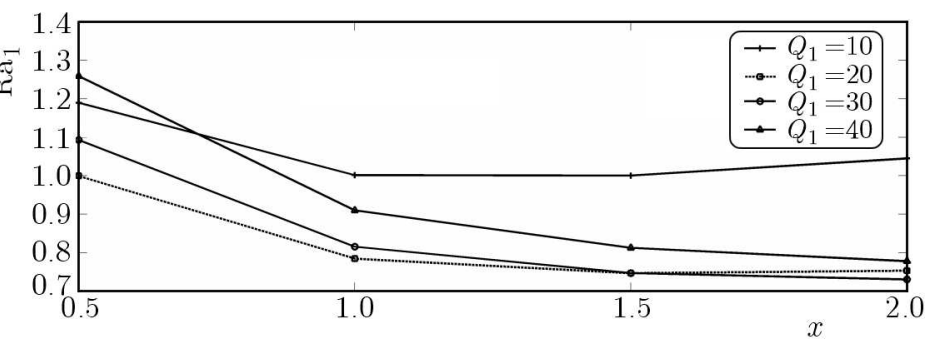

Fig. 3. Neural instability curve for different values of: (a) comprssibility parameter, $h(z)=5, P=5$, $\varepsilon=5, Q_{1}=10, Q_{M_{1}}=10, T_{A_{1}}=500$; (b) temperature gradient, $L=10, P=2, \varepsilon=3, Q_{1}=10$, $Q_{M_{1}}=10, T_{A_{1}}=500 ;$ (c) porosity, $L=5, h(z)=5, P=2, Q_{1}=20, Q_{M_{1}}=20, T_{A_{1}}=1000$;

(d) rotation parameter, $L=3, h(z)=3, P=3, \varepsilon=2, Q_{1}=50, Q_{M_{1}}=50$; (e) permeability, $L=2$, $h(z)=2, P=5, \varepsilon=3, Q_{1}=10, Q_{M_{1}}=10, T_{A_{1}}=50$; (f) magnetic field parameter, $L=5, h(z)=5$, $P=5, \varepsilon=5, Q_{M_{1}}=5, T_{A_{1}}=50$ 


\section{The overstable case}

Now, the possibility whether the instability may occur as overstability is examined. Equating the real and imaginary parts of equation (3.6) leads to

$$
\begin{aligned}
& {\left[(1+x)^{2}-\sigma_{i}^{2} E \operatorname{Pr}_{1} \operatorname{Pr}_{2}\right](1+x)=\operatorname{Ra}_{1} x \varepsilon P L h(z) \frac{(1+x) \varepsilon+\sigma_{i}^{2} P_{\operatorname{Pr}_{2}}}{\varepsilon^{2}+\sigma_{i}^{2} P^{2}}} \\
& \quad-P\left[(1+x) \varepsilon+\sigma_{i}^{2} \operatorname{Pr}_{1} P E\right] \frac{(1+x) Q_{1}+Q_{M_{1}} x}{\varepsilon^{2}+\sigma_{i}^{2} P^{2}} \\
& \quad-T_{A_{1}} P^{2}\left\{\left[(1+x)^{2}+\sigma_{i}^{2} \operatorname{Pr}_{2}\left(\operatorname{Pr}_{2}-\operatorname{Pr}_{1} E\right)\right](1+x)^{2}\right. \\
& \left.\quad+\left[(1+x)^{2}-\sigma_{i}^{2} \operatorname{Pr}_{2}\left(\operatorname{Pr}_{2}+2 \operatorname{Pr}_{1} E\right)\right] Q_{1} P(1+x)-\sigma_{i}^{4} E \operatorname{Pr}_{1} \operatorname{Pr}_{2}^{3}\right\} \\
& \sigma_{i}\left(\operatorname{Pr}_{2}+\operatorname{Pr}_{1} E\right)(1+x)^{2}=\operatorname{Ra}_{1} x \varepsilon P L h(z) \frac{\sigma_{i}\left[\operatorname{Pr}_{2} \varepsilon-P(1+x)\right]}{\varepsilon^{2}+\sigma_{i}^{2} P^{2}} \\
& \quad-\frac{\sigma_{i} P\left[\operatorname{Pr}_{1} E \varepsilon-P(1+x)\right]\left[(1+x) Q_{1}+Q_{M_{1}} x\right]}{\varepsilon^{2}+\sigma_{i}^{2} P^{2}}-T_{A_{1}} P^{2} \sigma_{i}\left[\left(\operatorname{Pr}_{2}+\operatorname{Pr}_{1} E\right)(1+x)^{3}\right. \\
& \left.\quad+Q_{1} P\left(2 \operatorname{Pr}_{2}+\operatorname{Pr}_{1} E\right)(1+x)^{2}+\sigma_{i}^{2} \operatorname{Pr}_{2}^{2}\left(\operatorname{Pr}_{2}+\operatorname{Pr}_{1} E\right)(1+x)-\sigma_{i}^{2} \operatorname{Pr}_{1} \operatorname{Pr}_{2}^{2} Q_{1} P E\right]
\end{aligned}
$$

Eliminating $\mathrm{Ra}_{1}$ between equations (5.1) and assuming $\sigma_{i}^{2}=y$, a four degree polynomial in $y$ is obtained as follows

$$
b_{0} y^{4}+b_{1} y^{3}+b_{2} y^{2}+b_{3} y+b_{4}=0
$$

where

$$
\begin{aligned}
b_{0}= & T_{A_{1}} P^{6} \operatorname{Pr}_{2}^{3}\left[\operatorname{PPr}_{2}(1+x)-\operatorname{Pr}_{1} E\left(\operatorname{Pr}_{2} \varepsilon+Q_{1} P^{2}\right)\right] \\
b_{4}= & T_{A_{1}} P^{3} \varepsilon^{4}(1+x)^{5}+\left[P \varepsilon^{4}+T_{A_{1}} P^{2} \varepsilon^{4}\left(\operatorname{Pr}_{1} E \varepsilon+Q_{1} P^{2}\right)\right](1+x)^{4} \\
& +\left[\operatorname{Pr}_{1} E \varepsilon^{5}+T_{A_{1}} Q_{1} P^{3} \varepsilon^{5}\left(\operatorname{Pr}_{1} E+\operatorname{Pr}_{2}\right)\right](1+x)^{3}+\left[Q_{1} P \varepsilon^{4}\left(\operatorname{Pr}_{1} E-\operatorname{Pr}_{2}\right)\right](1+x)^{2} \\
& +\left[Q_{M_{1}} P \varepsilon^{4} x\left(\operatorname{Pr}_{1} E-\operatorname{Pr}_{2}\right)\right](1+x)
\end{aligned}
$$

The coefficients $b_{1}, b_{2}$ and $b_{3}$ involving the large number of terms are not written here as they do not play any role in determining the overstability. Since $\sigma_{i}$ is real for overstability to occur, therefore all the roots of $y$ should be positive. So, from equation (5.2), the product of roots equals $b_{4} / b_{0}$ must be positive. $b_{0}$ is negative if

$$
P_{P_{2}}(1+x)<\operatorname{Pr}_{1} E\left(\operatorname{Pr}_{2} \varepsilon+Q_{1} P^{2}\right) \quad \text { i.e. } \quad P \kappa(1+x)<\eta E\left(\operatorname{Pr}_{2} \varepsilon+Q_{1} P^{2}\right)
$$

and $b_{4}$ is positive if

$$
\operatorname{Pr}_{1} E>\operatorname{Pr}_{2} \quad \text { i.e. } \quad \eta E>\kappa
$$

Thus, for inequalities (5.4) and (5.5), the overstability cannot occur and the principle of exchange of stabilities holds true. Therefore, the aforementioned inequalities are the sufficient conditions for the non-existence of overstability, violation of which does not necessarily imply the occurrence of overstability.

\section{Principal of exchange of stabilities and oscillatory modes}

Here, the conditions have been derived, if any, under which the principle of exchange of stabilities is satisfied and the possibility of oscillatory modes for the ferromagnetic fluid takes place. For this 
purpose, equation (3.2) is multiplied by $W^{*}$ (the complex conjugate of $W$ ) and then integrated over the range of $z$ using equations (3.3). With the help of boundary conditions (3.4), it gives

$$
\begin{aligned}
\left(\frac{\sigma}{\varepsilon}\right. & \left.+\frac{1}{P_{l}}\right) I_{1}-\left(g-\frac{\mu_{0} \chi M_{0} \nabla H}{\rho_{m} \alpha}\right) \frac{\kappa \alpha a^{2}}{\beta v L h(z)}\left(I_{2}+E \operatorname{Pr}_{1} \sigma^{*} I_{3}\right) \\
& +\frac{\mu_{0} M_{0}(1+\chi \Delta T)}{\rho_{m}} \frac{a^{2} \varepsilon}{\operatorname{Pr}_{2} H}\left(I_{4}+\operatorname{Pr}_{2} \sigma^{*} I_{5}\right) \\
& +\frac{\mu_{e} \varepsilon}{4 \pi \rho_{m} \operatorname{Pr}_{2}}\left(I_{6}+\operatorname{Pr}_{2} \sigma^{*} I_{4}\right)+d^{2}\left[\left(\frac{\sigma^{*}}{\varepsilon}+\frac{1}{P_{l}}\right) I_{7}+\frac{\mu_{e} \varepsilon}{4 \pi \rho_{m} \operatorname{Pr}_{2}}\left(I_{8}+\operatorname{Pr}_{2} \sigma^{*} I_{9}\right)\right]=0
\end{aligned}
$$

where the integrals $I_{1}-I_{9}$ are positive definite and defined as

$$
\begin{array}{lrl}
I_{1}=\int_{0}^{1}\left(|D W|^{2}+a^{2}|W|^{2}\right) d z & I_{2}=\int_{0}^{1}\left(|D \Theta|^{2}+a^{2}|\Theta|^{2}\right) d z & I_{3}=\int_{0}^{1}\left(|\Theta|^{2}\right) d z \\
I_{4}=\int_{0}^{1}\left(|D K|^{2}+a^{2}|K|^{2}\right) d z & I_{5}=\int_{0}^{1}|K|^{2} d z \\
I_{6}=\int_{0}^{1}\left(\left|D^{2} K\right|^{2}+a^{4}|K|^{2}+2 a^{2}|D K|^{2}\right) d z & I_{7}=\int_{0}^{1}\left(|Z|^{2}\right) d z \\
I_{8}=\int_{0}^{1}\left(|D X|^{2}+a^{2}|X|^{2}\right) d z & I_{9}=\int_{0}^{1}\left(|X|^{2}\right) d z
\end{array}
$$

Putting $\sigma=i \sigma_{i}$ in equation (6.1) and equating the imaginary part leads to

$$
\begin{gathered}
\sigma_{i}\left[\frac{I_{1}}{\varepsilon}+\left(g-\frac{\mu_{0} \chi M_{0} \nabla H}{\rho_{m} \alpha}\right) \frac{\kappa \alpha a^{2}}{\beta v L h(z)} \operatorname{Pr}_{1} E I_{3}-\frac{\mu_{0} M_{0}(1+\chi \Delta T)}{\rho_{m}} \frac{a^{2} \varepsilon}{\operatorname{Pr}_{2} H} \operatorname{Pr}_{2} I_{5}\right. \\
\left.-\frac{\mu_{e} \varepsilon \operatorname{Pr}_{2} I_{4}}{4 \pi \rho_{m} \operatorname{Pr}_{2}}-\frac{d^{2} I_{7}}{\varepsilon}+\frac{\mu_{e} \varepsilon d^{2} \operatorname{Pr}_{2} I_{9}}{4 \pi \rho_{m} \operatorname{Pr}_{2}}\right]=0
\end{gathered}
$$

From equation (6.3), it is concluded that either $\sigma_{i}=0$ or $\sigma_{i} \neq 0$, i.e. the modes may be non-oscillatory or oscillatory, respectively.

For a non-magneto-rotatory system (i.e. $I_{4}=I_{5}=I_{7}=0$ ), equation (6.2) reduces to

$$
\sigma_{i}\left[\frac{I_{1}}{\varepsilon}+\left(g-\frac{\mu_{0} \chi M_{0} \nabla H}{\rho_{m} \alpha}\right) \frac{\kappa \alpha a^{2}}{\beta v L h(z)} \operatorname{Pr}_{1} E I_{3}\right]=0
$$

It is obvious from equation (6.4) that if $g>\mu_{0} \chi M_{0} \nabla H /\left(\rho_{m} \alpha\right)$ then the term inside the square bracket will surely be positive, which leads to $\sigma_{i}=0$. Therefore, the modes are non-oscillatory and the principle of exchange of stabilities is satisfied. The oscillatory modes are introduced due to the presence of magnetic field and rotation. Thus the sufficient condition for the oscillatory modes to appear in the system is that the inequality $g<\mu_{0} \chi M_{0} \nabla H /\left(\rho_{m} \alpha\right)$ is satisfied.

Further, for an ordinary viscous fluid $\mu_{0}=0$ (i.e. $\Gamma=0$ ), equation (6.3) reduces to

$$
\sigma_{i}\left[\frac{I_{1}}{\varepsilon}+\frac{g \kappa \alpha a^{2}}{\beta v L h(z)} \operatorname{Pr}_{1} E I_{3}\right]=0
$$

which implies that $\sigma_{i}=0$ and the principles of exchange of stabilities is found to hold good. 


\section{Conclusions}

In this study, linear stability theory is used to find the critical Rayleigh number for the onset of both stationary and oscillatory thermal instabilities. The effects of various embedded parameters (rotation, magnetic field, compressibility, heat source, permeability and porosity) on thermal instability of a ferrofluid have been analyzed for the stationary state. The main conclusions drawn are presented as:

- For the case of stationary convection, compressibility, medium porosity and temperature gradient due to heat source (except at the lower boundary) accelerate the onset of convection, whereas rotation and ratio of magnetic permeability delay the onset of convection. The magnetic field and medium permeability have dual effects on thermal instability of the system, whereas in the absence of rotation, the stabilizing effect of the magnetic field and the destabilizing effect of the medium permeability is obvious from equations (3.16).

- The conditions $P \kappa(1+x)<\eta E\left(\operatorname{Pr}_{2} \varepsilon+Q_{1} P^{2}\right)$ and $\eta E>\kappa$ are the sufficient conditions for the non-existence of overstability. The principle of exchange of stabilities holds good for an ordinary viscous fluid and also in the absence of magnetic field and rotation for $g>\mu_{0} \chi M_{0} \nabla H /\left(\rho_{m} \alpha\right)$. Hence, the oscillatory modes are due to the presence of magnetic field and rotation only.

- Finally, from the present study, it is concluded that the compressibility, porosity, permeability, rotation, magnetic field and heat source parameter have profound effects on the onset of ferroconvection saturating a porous medium. The present work will also be useful for understanding more complex problems under different physical parameters mentioned above, and it is also possible to suppress the convective instability in a ferromagnetic fluid layer by controlling the magnitude of heat source, compressibility and medium porosity.

\section{References}

1. Aggarwal A.K., Makhija S., 2014, Hall effect on thermal stability of ferromagnetic fluid in porous medium in presence of horizontal magnetic field, Thermal Science, 18, 2, 503-514

2. BAILEy R.L., 1983, Lesser known applications of ferrofluids, Journal of Magnetism and Magnetic Materials, 39, 178-182

3. Berkovsky B.M., Bashtovoy V.G., 1996, Magnetic Fluids and Applications Handbook, Begell House, New York

4. Chandrasekhar S.C., 1981, Hydrodynamic and Hydromagnetic Stability, Dover, New York

5. FINLAYSON B.A., 1970, Convective instability in ferromagnetic fluids, Journal of Fluid Mechanics, 40, 4, 753-767

6. Hathaway D.B., 1979, Use of ferrofluid in moving coil loudspeakers, $d B$ Sound Engineering Magazine, 13, $42-44$

7. Kumar K., Singh V., Sharma S., 2014a, Thermo-magnetic convection in a rotating couplestress fluid through a Brinkman porous medium, International Journal of Applied Mathematics and Mechanics, 10, 8, 78-93

8. Kumar K., Singh V., Sharma S., 2014b, Magneto-rotational convection for ferromagnetic fluids in the presence of compressibility and heat source through a porous medium, Special Topics and Reviews in Porous Media, 5, 4, 311-323

9. Kumar K., Singh V., Sharma S., 2015, On the onset of convection in a dusty couple-stress fluid with variable gravity through a porous medium in hydromagnetics, Journal of Applied Fluid Mechanics, 8, 1, 55-63 
10. Lalas D.P., Carmi S., 1971, Thermoconvective stability of ferrofluids, Physics of Fluids, 14, 2, 436-437

11. McDonnel J.A.M., 1978, Cosmic Dust, John Wiley and Sons, Toronto

12. Moskowitz R., 1975, Dynamic sealing with magnetic fluids, ASLE Transactions, 18, 2, 135-143

13. Neuringer J.L., Rosensweig R.E., 1964, Ferrohydrodynamics, Physics of Fluids, 7, 12, 1927-1937

14. Newbower R.S., 1972, A new technique for circulatory measurements employing magnetic fluid tracers, Proceedings Biomedical Symposium, San Diego

15. Nield D.A., Bejan A., 2006, Convection in Porous Media, Springer, New-York

16. Odenbach S., 2002, Magnetoviscous Effects in Ferrofluids, Springer-Verlag, Berlin

17. Penfield P., Haus H.A., 1967, Electrodynamics of Moving Media, Institute of Technology Press, Cambridge

18. Rosensweig R.E., 1985, Ferrohydrodynamics, Cambridge University Press, Cambridge

19. Rosensweig R.E., 1987, Magnetic fluids, Review of Fluid Mechanics, 19, 437-463

20. Sharma R.C., 1997, Thermal instability of compressible fluid in the presence of rotation and magnetic field, Journal of Mathematical Analysis and Applications, 60, 227-235

21. Sherman A., Sutton G.W., 1962,Magnetohydrodynamic, Northwestern University Press, Illinois

22. Spiegal E.A., Veronis G., 1960, On the Boussinesq approximation for a compressible fluid, Astrophysical Journal, 131, 442-447

23. Sunil, Sharma D., Sharma R.C., 2005a, Effect of dust particles on thermal convection in ferromagnetic fluid saturating a porous medium, Journal of Magnetism and Magnetic Materials, 288, 183-195

24. Sunil, Sharma D., Sharma V., 2005b, Effect of dust particles on rotating ferromagnetic fluid heated from below saturating a porous medium, Journal of Colloid and Interface Science, 291, 152-161 